\title{
Educação Alimentar e Nutricional em Grupo: Caracterização Socioeconômica, Consumo Alimentar e Estado Nutricional dos Participantes
}

\author{
Isabella dos Santos Araújo de Oliveira, ${ }^{1}$ Alice Helena de Souza Paulino, ${ }^{2}$ \\ Tábatta Renata de Pereira Brito, ${ }^{1}$ Bruno Martins Dala-Paula ${ }^{3}$
}

\section{RESUMO}

Ações de educação em saúde em grupo apresentam vantagens em relação às intervenções individualizadas. A educação alimentar e nutricional realizada em grupos possui baixo custo de operacionalização, ampla abrangência, contribui com o fortalecimento de redes sociais, além de ser um método efetivo de promoção da saúde. É necessário conhecer, no entanto, os participantes, a fim de que as intervenções possam ser orientadas. Nesse sentido, o objetivo deste estudo foi caracterizar o perfil socioeconômico, padrão de consumo alimentar e o estado nutricional de participantes de uma ação de educação alimentar e nutricional em grupo. Trata-se de um estudo seccional realizado com 26 indivíduos matriculados em uma Universidade Aberta à Pessoa Idosa. Os dados foram obtidos por meio de questionários socioeconômicos, inquéritos dietéticos e avaliação antropométrica. Os resultados indicaram que a população estudada apresenta renda mensal e escolaridade superior à média da população brasileira, elevada frequência de inadequação quanto à distribuição dos macronutrientes, em especial de lipídios $(n=13)$, elevada frequência de ingestão aumentada de ácidos graxos saturados $(n=16)$, trans $(n=14)$ e de sódio $(n=9)$, elevado número de participantes consumindo fibras alimentares $(n=18)$ e cálcio abaixo $(n=23)$ do preconizado, e inadequação para a ingestão de vitamina $D(n=26)$. Houve predomínio de sobrepeso, risco cardiovascular e frequência acima de 35\% de situação de atenção para a redução de massa muscular. A realização desta pesquisa permitiu entender o contexto social dos participantes e identificar as necessidades nutricionais, norteando a escolha de temas e metodologias para as ações de educação alimentar e nutricional em grupo.

Palavras-chave: promoção da saúde; educação em saúde; educação alimentar e nutricional; consumo de alimentos; estado nutricional.

FOOD AND NUTRITION EDUCATION IN GROUP:

SOCIOECONOMIC CHARACTERIZATION, FOOD CONSUMPTION AND NUTRITIONAL STATUS OF PARTICIPATS

\section{ABSTRACT}

Health education in group have advantages over individualized interventions. Food and nutrition education carried out in groups has low operating costs, wide coverage, contributes to the strengthening of social networks, in addition to be an effective method of health promotion. However, it is necessary to know the participants, so that interventions can be targeted. In this sense, the objective of this study was to characterize the socioeconomic profile, pattern of food consumption and the nutritional status of participants in a group of food and nutrition education. This is a cross-sectional study conducted with 26 individuals enrolled in a University Open to the Elderly. The data were obtained through socioeconomic questionnaires, dietary surveys, and anthropometric assessment. The results indicated that the studied population has a monthly income and education higher than the average of the Brazilian population, a high frequency of inadequacy regarding the distribution of macronutrients, especially lipids ( $n=13$ ); high frequency of increased intake of saturated $(n=16)$ and trans $(n=14)$ fatty acids, and sodium $(n=9)$. High number of participants consuming dietary fiber $(n=18)$ and calcium below $(n=23)$ the recommended level; and inadequacy for vitamin $D(n=26)$ intake. There was a predominance of overweight, cardiovascular risk and a frequency above $35 \%$ of attention to the reduction of muscle mass. The realization of this research allowed to understand the social context of the participants and to identify the nutritional needs, guiding the choice of themes and methodologies for the actions of food and nutrition education in group.

Keywords: health promotion; health education; food and nutrition education; food consumption; nutritional status.

RECEBIDO EM: 19/5/2020

MODIFICAÇÕES SOLICITADAS EM: 12/4/2021

ACEITO EM: 19/4/2021

\footnotetext{
$\overline{1}$ Universidade Federal de Alfenas (Unifal). Alfenas/MG, Brasil.

2 Universidade Federal de Alfenas (Unifal). Alfenas/MG, Brasil.

${ }^{3}$ Autor correspondente. Universidade Federal de Alfenas (Unifal). R. Gabriel Monteiro da Silva, 700 - Centro. Alfenas/MG, Brasil. CEP 37130-001. http://lattes.cnpq.br/5246931390431639. https://orcid.org/0000-0002-5022-4796. bruno.paula@ unifal-mg.edu.br
} 


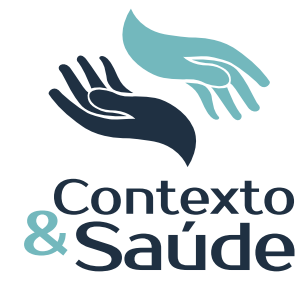

\section{INTRODUÇÃO}

O envelhecimento populacional é uma realidade no Brasil, e o aumento do número de idosos na população leva ao surgimento de novas demandas na área da saúde relacionadas à maior incidência de doenças crônicas e, consequentemente, maior e mais prolongado uso de serviços de saúde (OMS, 2015). Dentre as diversas ações de prevenção dessas doenças, destaca-se a educação em saúde, definida pelo Ministério da Saúde como o processo educativo de construção de conhecimentos em saúde, que contribui para aumentar a autonomia das pessoas no seu cuidado (BRASIL, 2006).

Ações de educação em saúde em grupo apresentam vantagens em relação às intervenções individualizadas e, em virtude disso, a organização de grupos para intervenções coletivas, especialmente no que se refere à educação alimentar e nutricional (EAN), tem sido cada vez mais frequentes. Tais vantagens incluem o aprimoramento do processo de trabalho, a racionalização de recursos financeiros, o alcance de maior número de indivíduos, a diminuição da espera por atendimentos individuais, a participação ativa dos indivíduos no processo educativo e a maior interação entre a equipe de profissionais de saúde e usuários dos serviços de saúde (BEDESCHI et al., 2018). Além disso, as atividades em grupo podem ajudar a promover troca de vivências a respeito de dificuldades enfrentadas e experiências adquiridas, assim como podem contribuir para diminuir isolamento social (SOEIRO et al., 2019).

Considerando-se a importância de estratégias de educação em saúde cujo objetivo é melhorar a alimentação, a nutrição e a saúde da população, ações de EAN têm sido fortemente estimuladas (VINCHA; BOGUS; CERVATO-MANCUSO, 2020). Um estudo realizado com um grupo de 50 idosos americanos observou que, após 12 semanas de intervenção nutricional, houve aumento no consumo de frutas e vegetais, além de diminuição do consumo de gordura, indicando que intervenções dessa natureza podem ser uma maneira de baixo custo para meIhorar comportamentos alimentares entre idosos, afetando, significativamente, a saúde dessa população (SCHALAFF et al., 2018).

Queiroz et al. (2020) realizaram uma pesquisa on-line, sobre o tema "saúde", com uma população de 1.810 brasileiros adultos, e registraram a participação predominante de mulheres $(74,87 \%)$, indicando possível relação da maior preocupação deste grupo com a saúde em geral. Ainda sobre esta pesquisa, $53,6 \%$ dos participantes possuíam idade superior a 40 anos, com alto nível de escolaridade, sendo $48,72 \%$ graduados, com renda mensal familiar superior a $\mathrm{R} \$ 5.000,00$. Os pesquisadores associaram as características sociodemográficos com o elevado percentual de participantes que reportou consumir frutas e hortaliças em cinco ou mais dias durante a semana, equivalente a 50,3\% e 66,1\%, respectivamente. Há registros na literatura sobre a maior aderência aos hábitos alimentares saudáveis, tendo em vista as recomendações do Guia Alimentar para a População Brasileira, por indivíduos com maior idade quando comparados aos de menor idade. Além disso, a região de residência, considerando as grandes regiões brasileiras, também foi percebida como variável determinante para a aderência às práticas alimentares saudáveis (GABE; JAIME, 2020).

Editora Unijuí - Revista Contexto \& Saúde - ISSN 2176-7114 - v. 21, n. 43, jul./set. 2021 
A avaliação das características sociodemográficas dos participantes e o padrão alimentar de consumo são de suma importância para o direcionamento de ações de educação em saúde assertivas, em consonância com a realidade e anseios dos participantes (VINCHA; BOGUS; CERVATO-MANCUSO, 2020). Desse modo, este estudo objetivou caracterizar o perfil socioeconômico, padrão de consumo alimentar e o estado nutricional de participantes de uma ação de educação alimentar e nutricional em grupo. O conhecimento sistematizado das características dos indivíduos que integram um grupo possibilita a adoção de estratégias mais eficazes e efetivas, assim como a seleção de temas mais relevantes à promoção da saúde, visando a suprir as demandas identificadas.

\section{MÉTODO}

Trata-se de um estudo quantitativo com delineamento seccional descritivo das características, sociais, econômicas, educacionais, antropométricas, nutricionais e hábitos alimentares de um grupo de adultos maduros e idosos residentes no município de Alfenas, MG.

Participaram do estudo indivíduos com idade de 50 anos ou mais matriculados no Programa de Extensão "Universidade Aberta à Pessoa Idosa" (Unapi) e participantes da ação componente "Grupo: Viver Bem a Nova Idade", registrada na Pró-Reitoria de Extensão da Universidade Federal de Alfenas (Unifal-MG). Todos os matriculados na referida ação em 2018, foram convidados a participar da pesquisa, contabilizando uma amostra não probabilística por conveniência de 26 indivíduos. O "Grupo Viver Bem a Nova Idade" tinha como propósito a realização de encontros semanais com um grupo de adultos maduros e idosos para a realização de diferentes atividades de educação alimentar e nutricional e proporcionar a socialização dos envolvidos.

A avaliação dos participantes foi realizada após submissão e aprovação pelo Comitê de Ética em Pesquisa da Unifal-MG (CAAE: 88358318.8.0000.5142; parecer no 2.668.975). Os critérios de inclusão para participação na pesquisa foram: idade superior a 50 anos; condições neurológicas e cognitivas adequadas para responder os questionários; e a assinatura do Termo de Consentimento Livre e Esclarecido (TCLE).

A caracterização do perfil social, econômico e educacional dos participantes foi realizada a partir da aplicação de um questionário semiestruturado por uma equipe treinada de discentes do curso de Nutrição da Unifal-MG.

A caracterização dos hábitos alimentares dos participantes foi realizada a partir de um questionário estruturado e por dois inquéritos dietéticos, um Recordatório Alimentar de 24 horas (R24) e um Questionário de Frequência do Consumo Alimentar (QFCA). Os alimentos e preparações informados no R24h foram convertidos de medida caseira para peso, em gramas, a partir da Tabela de Composição Química dos Alimentos e Medidas Caseiras (PACHECO, 2011). A energia, carboidratos, fibras alimentares, proteínas, lipídeos totais e frações (ácidos graxos saturados, insaturados - monoinsaturados, poli-insaturados e trans - e colesterol), cálcio, sódio e vitamina D consumidos, foram calculados a partir do software NDSR ${ }^{\circ}$. O consumo de energia e dos nutrientes supracitados foram

Editora Unijuí - Revista Contexto \& Saúde - ISSN 2176-7114 - v. 21, n. 43, jul./set. 2021 
comparados conforme indicado na Tabela 1, respeitando as características de cada participante, como faixa etária, sexo e estado nutricional, quando mencionado.

Tabela 1 - Recomendações e referências de energia e nutrientes para adultos maduros e idosos

\begin{tabular}{|c|c|c|}
\hline Energia e nutrientes & Recomendação de consumo diário & Referência \\
\hline \multirow{2}{*}{ Energia } & - Cálculo de necessidade energética estimada. & IOM (2005) \\
\hline & - $30 \mathrm{kcal} / \mathrm{kg}$ de peso corpóreo para idosos* & ESPEN (2019) \\
\hline Carboidratos & - Faixa de distribuição aceitável (45-65\% VET) & IOM (2005) \\
\hline \multirow[t]{2}{*}{ Fibras alimentar } & $\begin{array}{l}\text { - Ingestão adequada (AI), mulheres > } 51 \text { anos: } \\
21 \text { g; homens > } 51 \text { anos: } 30 \text { g) }\end{array}$ & IOM (2005) \\
\hline & • $25 \mathrm{~g}$ (Mulheres e homens idosos) & ESPEN (2019) \\
\hline \multirow[t]{2}{*}{ Proteínas } & - Faixa de distribuição aceitável (10-35\% VET) & IOM (2005) \\
\hline & $\bullet>1 \mathrm{~g} / \mathrm{kg}$ de peso corpóreo para idosos** & ESPEN (2019) \\
\hline Lipídeos totais & Faixa de distribuição aceitável (20-35\% VET) & IOM (2005) \\
\hline AG Saturado & $\bullet<10 \%$ VET & FAO/WHO (2009) \\
\hline Mufa & •15-20\% VET conforme ingestão de lipídeos & FAO/WHO (2009) \\
\hline Pufa & $\bullet 6-11 \%$ VET & FAO/WHO (2009) \\
\hline AG trans & $\bullet<1 \%$ VET & FAO/WHO (2009) \\
\hline Vitamina D & $\begin{array}{l}\text { - EAR (10 mg para mulheres e homens > } 51 \\
\text { anos) }\end{array}$ & IOM (2011) \\
\hline Cálcio & $\begin{array}{l}\text { - EAR (800 mg para homens entre 51-70 anos } \\
\text { e } 1.000 \text { mg para mulheres }>51 \text { anos) }\end{array}$ & IOM (2011) \\
\hline Sódio & $\bullet>\mathrm{Al}$ (1.500 mg) e < UL (2.300 mg) & IOM (2019) \\
\hline
\end{tabular}

Leg.: VET: valor energético total; EAR: necessidade média estimada; AG: ácidos graxos; Mufa: ácidos graxos monoinsaturados; Pufa: ácidos graxos poli-insaturados; UL: nível máximo de ingestão tolerável; * Conforme recomendação do Espen foi adotado o seguinte ajuste: 29, 28 e $27 \mathrm{kcal} / \mathrm{kg}$ para indivíduos com IMC entre 27$30,30-33$ e $>33 \mathrm{~kg} / \mathrm{m}^{2}$, respectivamente. ${ }^{* *}$ Conforme recomendação do Espen foi adotado o seguinte ajuste: $1,5 \mathrm{~g} / \mathrm{kg}$ para indivíduos com IMC $<20,0 \mathrm{~kg} / \mathrm{m}^{2}$ (ESPEN, 2019).

Fonte: Autoria própria.

Para reduzir a interferência do valor energético total em relação ao consumo nutricional, todas as análises foram realizadas pelo método da densidade de nutriente, após ajuste de cada nutriente para $1.000 \mathrm{kcal}$, assim como das referências de comparação. Com exceção da vitamina, expressa em mcg/1.000 kcal, do cálcio e sódio, expressos em mg/1.000 kcal, todos os demais nutrientes, fibra e componentes lipídicos foram expressos em g/1.000 kcal.

O QFCA era do tipo semiquantitativo, contendo 28 alimentos. A quantidade e a frequência de consumo relatada para cada item foi convertida em porções, sendo a frequência transformada para consumo diário, dividindo as porções semanais e mensais por 7 e 30, respectivamente. Os alimentos do QFCA foram reunidos em grupos de alimentos (BRASIL, 2014) e a frequência de con- 
sumo diário de porções foi utilizada a fim de possibilitar a comparação com as recomendações do Ministério da Saúde (BRASIL, 2006, 2009).

As aferições das medidas antropométricas foram realizadas conforme indicado pelo Sistema de Vigilância Alimentar e Nutricional (Sisvan) do Ministério da Saúde (BRASIL, 2011). A classificação do estado nutricional foi realizada com base no índice de massa corporal segundo as recomendações do Sisvan, que considera as modificações na composição corporal próprias do envelhecimento, sendo o estado nutricional classificado em baixo peso (IMC $\leq 18,5$ e IMC $\leq 22$ $\mathrm{kg} / \mathrm{m}^{2}$ ), adequado ou eutrófico (IMC $\geq 18,5$ e $<25 \mathrm{~kg} / \mathrm{m}^{2}$ e IMC $\geq 22$ e $<27 \mathrm{~kg} /$ $\mathrm{m}^{2}$ ), sobrepeso (IMC $\geq 25 \mathrm{e}<30 \mathrm{~kg} / \mathrm{m}^{2}$ e IMC $>27 \mathrm{~kg} / \mathrm{m}^{2}$ ) para adultos maduros (participantes com idade entre 50 e 60 anos) e idosos (acima de 60 anos), respectivamente. Para os adultos maduros foi também considerada a classificação em obesos (IMC $\geq 30 \mathrm{~kg} / \mathrm{m}^{2}$ ) (BRASIL, 2011).

O risco para doenças cardiovasculares foi avaliado por meio da medida da circunferência da cintura de acordo com os valores propostos pela Organização Mundial da Saúde, a qual indica risco para indivíduos com valores de circunferência de cintura (CC) de $\geq 80 \mathrm{~cm}$ para mulheres e $\geq 94 \mathrm{~cm}$ para homens (OMS, 1998). Nos participantes idosos, o risco de sarcopenia e redução da massa muscular foi avaliado a partir da circunferência de cintura, levando em consideração os seguintes pontos de corte: circunferência de panturrilha de $\leq 34 \mathrm{~cm}$ como indicativo de atenção para a redução de massa magra, e $\leq 31$ como risco de sarcopenia (BRASIL, 2017).

Os dados foram digitados no programa Excel e as análises de distribuições de frequências, médias e desvios-padrão, foram realizadas no software Stata versão 13.0.

\section{RESULTADOS E DISCUSSÕES}

\section{Análise do perfil socioeconômico e educacional}

Dos 31 matriculados no projeto de extensão "Viver Bem a Nova Idade" em 2018, 26 concordaram em participar da pesquisa, assinando o TCLE. Cerca de $92 \%$ eram do sexo feminino $(n=24)$ e $8 \%$ do sexo masculino $(n=2)$. O grupo apresentou predomínio de indivíduos idosos em relação aos adultos maduros (Tabela 1), sendo a média de idade do grupo equivalente a 63,5 anos (desvio padrão = 7,3 anos).

Os participantes desta pesquisa são, em sua maioria, da raça branca, seguida da parda. Não foram registradas respostas para as demais raças, presentes como opção no questionário (negra, indígena, amarela ou outra). 
Tabela 1 - Características sociais, econômicas e educacionais dos participantes do Grupo Viver Bem a Nova Idade, Alfenas/MG, 2018

\begin{tabular}{|c|c|c|c|c|c|}
\hline \multirow[t]{2}{*}{ Características } & \multicolumn{2}{|c|}{ Adultos maduros $(n=7)$} & \multicolumn{2}{|c|}{ Idosos $(n=19)$} & \multirow{2}{*}{ Tota } \\
\hline & Frequência & $\%$ & Frequência & $\%$ & \\
\hline \multicolumn{6}{|l|}{ Sexo } \\
\hline Masculino & 1 & 14,3 & 1 & 5,3 & 2 \\
\hline Feminino & 6 & 85,7 & 18 & 94,7 & 24 \\
\hline \multicolumn{6}{|l|}{ Raça } \\
\hline Branco & 4 & 57,1 & 17 & 89,5 & 21 \\
\hline Pardo & 2 & 28,6 & 2 & 10,5 & 4 \\
\hline NSR/NR & 1 & 14,3 & 0 & 0 & 1 \\
\hline \multicolumn{6}{|l|}{ Estado civil } \\
\hline Solteiro & 0 & 0 & 5 & 26,3 & 5 \\
\hline Casado & 6 & 85,7 & 5 & 26,3 & 11 \\
\hline Divorciado ou separado & 1 & 14,3 & 3 & 15,8 & 4 \\
\hline Viúvo & 0 & 0 & 6 & 31,6 & 6 \\
\hline \multicolumn{6}{|l|}{ Trabalho remunerado } \\
\hline Sim & 1 & 14,3 & 4 & 21,1 & 5 \\
\hline Não & 5 & 71,4 & 14 & 73,7 & 19 \\
\hline NSR/NR & 1 & 14,3 & 1 & 5,3 & 2 \\
\hline \multicolumn{6}{|l|}{ Aposentados } \\
\hline Sim & 4 & 57,1 & 16 & 84,2 & 20 \\
\hline Não & 3 & 42,9 & 3 & 15,8 & 6 \\
\hline \multicolumn{6}{|l|}{ Rendimento individual (R\$) } \\
\hline$\leq 908,00$ & 0 & 0 & 2 & 10,5 & 2 \\
\hline $908,00-2.862,00$ & 5 & 71,4 & 9 & 47,4 & 14 \\
\hline$>2.862,00-5.724,00$ & 1 & 14,3 & 6 & 31,6 & 7 \\
\hline$>5.724,00$ & 1 & 14,3 & 1 & 5,3 & 2 \\
\hline \multicolumn{6}{|l|}{ Rendimento familiar (R\$) } \\
\hline$\leq 908,00$ & 0 & 0 & 0 & 0 & 0 \\
\hline $908,00-2.862,00$ & 3 & 42,9 & 5 & 26,3 & 8 \\
\hline$>2.862,00-5.724,00$ & 3 & 42,9 & 6 & 31,6 & 9 \\
\hline$>5.724,00$ & 1 & 14,3 & 3 & 15,8 & 4 \\
\hline NSR/NR & 0 & 0 & 5 & 26,3 & 5 \\
\hline \multicolumn{6}{|l|}{ Número de filhos } \\
\hline Nenhum & 2 & 28,6 & 5 & 26,3 & 7 \\
\hline 1 & 2 & 28,6 & 5 & 26,3 & 7 \\
\hline 2 a 3 & 2 & 28,6 & 9 & 47,4 & 11 \\
\hline 4 & 1 & 14,3 & 0 & 0 & 1 \\
\hline \multicolumn{6}{|c|}{$\begin{array}{l}\text { Número de moradores na re- } \\
\text { sidência }\end{array}$} \\
\hline 1 (mora só) & 0 & 0 & 4 & 21,1 & 4 \\
\hline 2 & 4 & 57,1 & 9 & 47,1 & 13 \\
\hline Acima de 3 & 3 & 42,9 & 6 & 31,6 & 9 \\
\hline \multicolumn{6}{|l|}{ Escolaridade } \\
\hline Fundamental & 1 & 14,3 & 7 & 36,8 & 8 \\
\hline Médio & 1 & 14,3 & 5 & 26,3 & 6 \\
\hline Superior & 3 & 42,9 & 5 & 26,3 & 8 \\
\hline Pós-Graduação & 2 & 28,6 & 2 & 10,5 & 4 \\
\hline \multicolumn{6}{|l|}{ Possui horta na residência } \\
\hline $\operatorname{sim}$ & 4 & 57,1 & 11 & 57,9 & 15 \\
\hline Não & 3 & 42,9 & 8 & 42,1 & 11 \\
\hline
\end{tabular}

Legenda: NSR/NR: não soube responder/não respondeu.

Fonte: Autoria própria. 


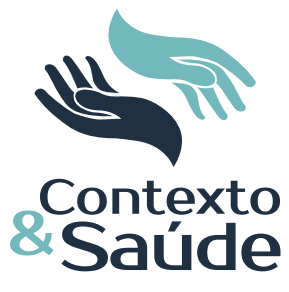

A maioria dos participantes adultos maduros estavam casados no momento da entrevista, enquanto grande parte dos idosos autodeclaram-se viúvos. A maioria do grupo não realizava trabalho remunerado, mas eram aposentados, possuindo rendimento mensal individual entre $\mathrm{R} \$ 908,00$ e $\mathrm{R} \$ 2.862,00$ e familiar entre $R \$ 2.862,00$ e $R \$ 5.724,00$. O rendimento mensal familiar de todos os participantes desta pesquisa foi superior a $R \$ 908,00$, enquanto $22,0 \%$ das famílias brasileiras possuem renda mensal inferior a essa quantia. Esses dados excluem todos os entrevistados desta pesquisa da situação de pobreza extrema, caracterizado por renda diária individual inferior a US\$1,90. A literatura apresenta clara e direta associação entre a densidade de nutrientes da dieta com os diferentes estratos socioeconômicos da população (CANO-IBÁÑEZ et al., 2020), além de mostrar que o consumo mais frequente de frutas e hortaliças (igual ou superior a cinco dias por semana) tem sido prevalente em uma população participante de uma pesquisa, quando se constatou predomínio dos respondentes com renda familiar mensal igual ou superior a R\$5.000,00 (QUEIROZ et al., 2020).

Dos 26 participantes, apenas 7 não possuem filhos e 4 relataram viver só. A maioria dos participantes relatou estar casado ou viver em companhia de outra pessoa em sua residência, o que é considerado um forte preditor para uma dieta com elevada qualidade nutricional. Além disso, Cano-lbáñez et al. (2020) verificaram, a partir de um estudo transversal com 6.646 participantes espanhóis, associação negativa entre a condição de morar só e a motivação para adoção de uma dieta saudável.

Todos os entrevistados eram alfabetizados, sendo o nível de escolaridade do grupo heterogêneo. Assim como a renda, a escolaridade também influencia nas escolhas alimentares. Um estudo, a partir de dados da Pesquisa Nacional de Saúde realizada pelo Instituto Brasileiro de Geografia e Estatística (IBGE), entrevistou 60.202 participantes de 18 anos ou mais, e constatou que indivíduos com níveis de menor escolaridade apresentam consumo de carne com maior teor de gordura e consumo insuficiente de vegetais de folhas, vegetais crus e frutas (BARROS et al., 2016). A literatura aponta o elevado nível educacional como um significante preditor para dietas com elevada densidade de micronutrientes (BRASIL et al., 2019; CANO-IBÁÑEZ et al., 2020; QUEIROZ et al., 2020).

Mais da metade dos participantes relataram possuir horta na residência, o que pode contribuir com o acesso facilitado aos alimentos in natura vegetais. O Ministério da Saúde do Brasil preconiza que uma alimentação saudável deva possuir, como base, alimentos in natura ou minimamente processados de origem vegetal e baixa representatividade de alimentos ultraprocessados (BRASIL, 2014).

\section{Análise do perfil alimentar e do consumo energético e nutricional}

O consumo energético médio diário dos participantes do Grupo Viver Bem a Nova Idade, agrupados conforme classificação etária em adultos maduros e idosos, foi inferior às necessidades estimadas pelo Institute of Medicine (IOM, 2005) em 38,6\% e 36,5\%, e pelo European Society for Clinical Nutrition and Metabolism (ESPEN, 2019) em 17,9\% e 21,6\%, respectivamente (Figura 1).

Editora Unijuí - Revista Contexto \& Saúde - ISSN 2176-7114 - v. 21, n. 43, jul./set. 2021 
Figura 1 - Comparação do consumo médio de energia (kcal) pelos participantes do Grupo Viver Bem a Nova Idade, agrupados conforme classificação etária, com as necessidades energéticas estimadas pelo Institute of Medicine (IOM, 2005) e European Society for Clinical Nutrition and Metabolism (ESPEN, 2019).

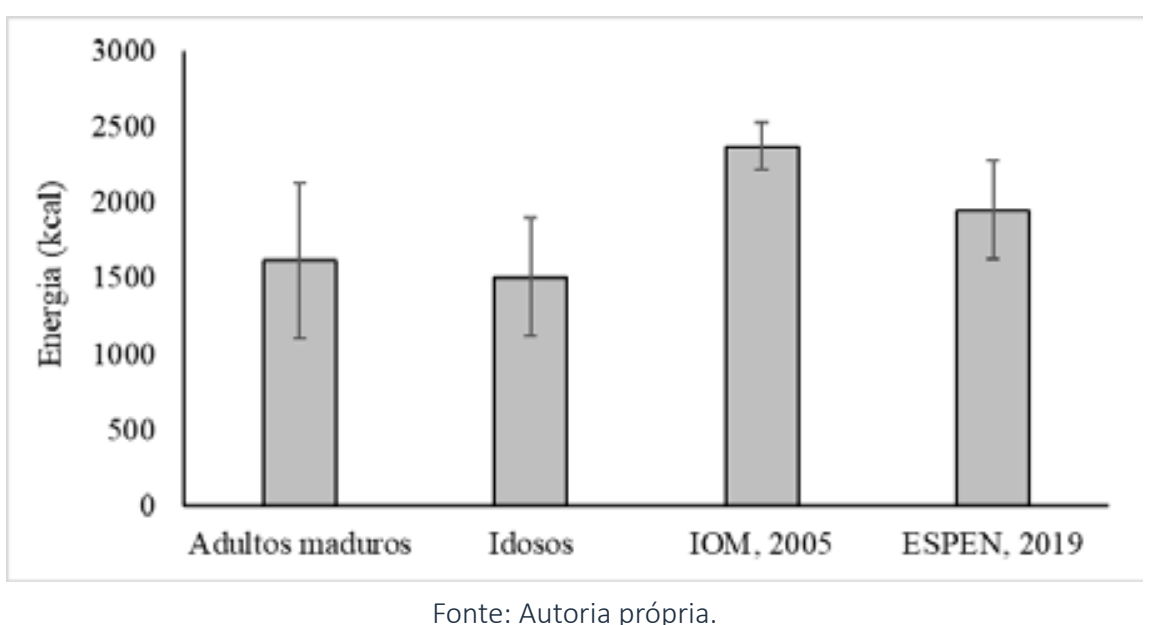

Dietas restritivas para perda de peso devem ser evitadas em idosos, mesmo aqueles com diagnóstico de sobrepeso, a fim de se prevenir a perda de massa muscular acompanhada de declínio funcional, aumento da prevalência de sarcopenia, fragilidade, ocorrência de fraturas e desnutrição. Essa conduta é justificada pela tendência aumentada dos idosos de recuperar o peso perdido após a intervenção dietética restritiva em massa gorda e não em tecido muscular. A repetição do ciclo de perda e ganho de peso em idosos está associada à obesidade sarcopênica. Sendo assim, intervenções para a perda de peso em idosos devem ser balanceadas, e as necessidades energéticas mínimas não devem ser inferiores a $27 \mathrm{kcal} / \mathrm{kg}$ de peso corpóreo e associadas, sempre que possível, à prática de atividade física monitorada (ESPEN, 2019).

Considerando as recomendações nutricionais, houve maior frequência de adequação $(<80 \%)$ para o consumo de proteínas entre adultos maduros e idosos, tendo como base o intervalo aceitável de distribuição deste nutriente definido pelo IOM (2005) (Tabela 2). Mesmo utilizando como referência a recomendação proposta pela Espen (2019), que leva em consideração o peso corpóreo de cada indivíduo, a taxa de adequação de consumo permaneceu elevada quando comparada com a observada para os demais nutrientes, sendo de $85,1 \%$ $\pm 31,5 \%$ e $95,3 \% \pm 41,8 \%$ para os participantes adultos maduros e idosos, respectivamente.

O grupo apresentou elevada frequência de consumo abaixo da Al para fibras alimentares, consumo abaixo das recomendações de ácidos graxos mono e poli-insaturados e consumo insuficiente de vitamina $D$ e cálcio para adultos maduros e idosos. O baixo consumo de fibra alimentar está associado à maior prevalência de constipação intestinal e de câncer intestinal (GIANFREDI et al., 2019). O consumo insuficiente de ácidos graxos mono e poli-insaturados está associado ao aumento de risco para diabetes mellitus, aumento da prevalência de doenças coronarianas e alguns tipos de câncer. Dietas com teores adequados desses ácidos graxos são recomendadas a fim de reduzir o risco de síndrome me- 
tabólica (FAO/WHO, 2009). Yang et al. (2020) recomendam evitar a deficiência de vitamina $D$ e o sedentarismo, a fim de reduzir perdas musculares e prevenir a sarcopenia.

Em contrapartida, os resultados demonstraram elevada frequência de indivíduos com consumo excessivo de lipídeos totais, considerando o intervalo aceitável de distribuição deste macronutriente; ácidos graxos saturados e trans, levando em conta os limites máximos aceitáveis; e sódio, considerando o nível máximo de ingestão tolerável desse elemento (Tabela 2). O desequilíbrio na distribuição de macronutrientes, a exemplo do consumo elevado de lipídeos, aumenta a densidade energética da alimentação e pode estar associado com elevada ingestão de ácidos graxos saturados. O consumo elevado de ácidos graxos saturados e insaturados trans eleva os níveis séricos de LDL colesterol e, consequentemente, aumenta a prevalência de doenças cardiovasculares. A substituição de ácidos graxos saturados por mono e poli-insaturados e a redução da ingestão de gorduras trans, apresentam forte evidência científica no controle dos níveis de LDL colesterol e redução da prevalência de doenças cardiovasculares (AHA, 2017). O consumo alimentar de sódio deve ser controlado por meio da redução da utilização de sal de cozinha e temperos industrializados, e o consumo de alimentos ultraprocessados deve ser evitado (BRASIL, 2014). O excesso de sódio dietético contribui com a alta da pressão arterial, aumentando, assim, a prevalência de hipertensão arterial. Dados da pesquisa Vigitel, realizada em 2018, indicaram que $24,7 \%$ da população que vive nas capitais relataram ter diagnóstico de hipertensão arterial, posto que $49,5 \%$ das pessoas com idade entre 55 e 64 anos e $60,9 \%$ dos idosos, com idade igual ou superior a 65 anos, são hipertensos (BRASIL, 2019). 

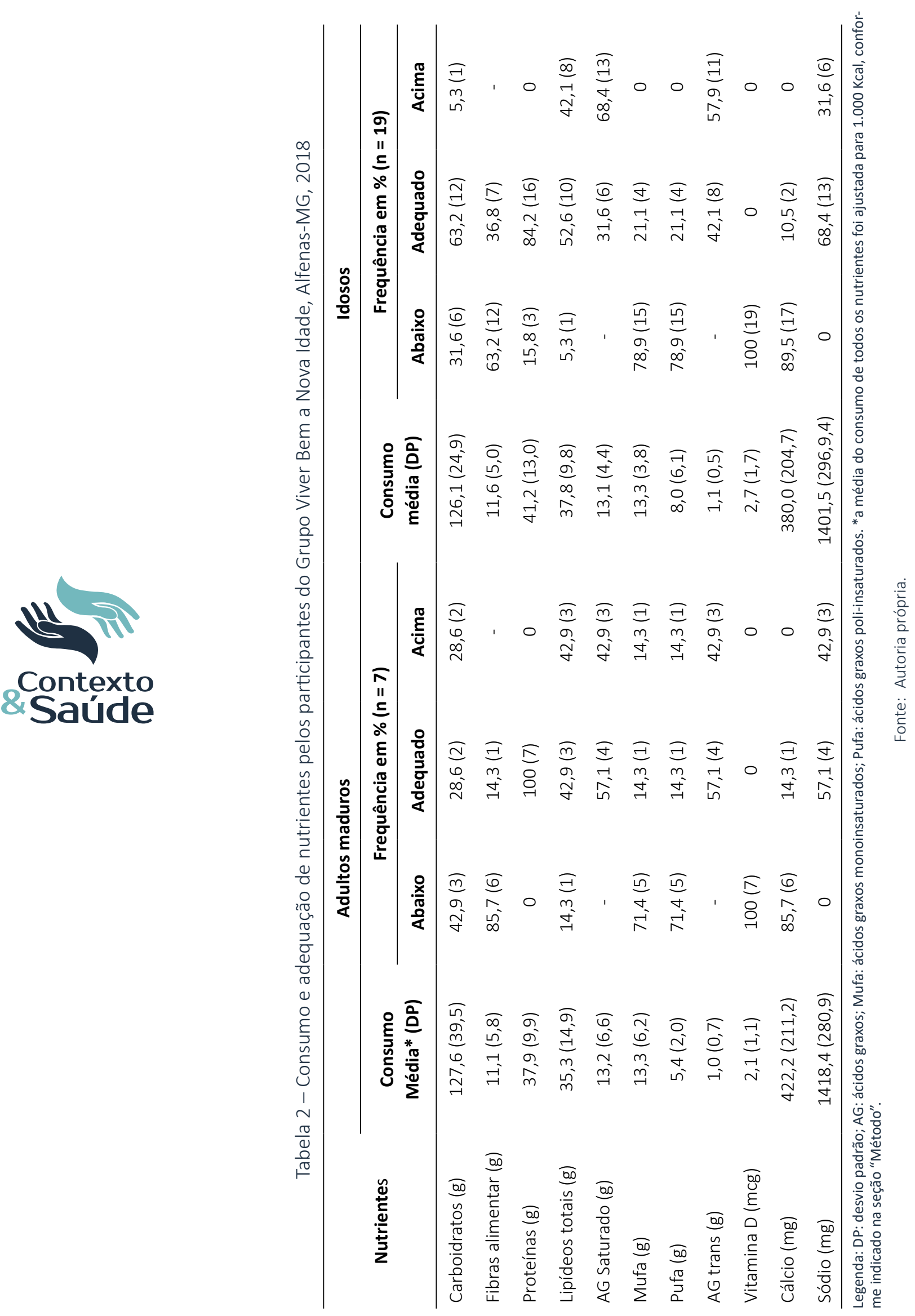

Editora Unijuí - Revista Contexto \& Saúde - ISSN 2176-7114 - v. 21, n. 43, jul./set. 2021 


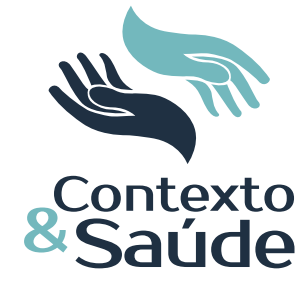

O perfil do consumo dos grupos de alimentos, obtido a partir da aplicação do QFCA, demonstrou consumo abaixo do recomendado pelo Ministério da Saúde (BRASIL, 2006, 2009) para os itens do grupo dos pães, cereais e massas, e de frutas, leguminosas e leite e derivados (Tabela 3). A utilização de QFCA validado para a população estudada é extremamente importante para a obtenção de resultados mais próximos do consumo real dos entrevistados. Este estudo utilizou um QFCA não validado, podendo a frequência do consumo alimentar encontrada estar subestimada ou superestimada. Resultados encontrados pela aplicação dos inquéritos dietéticos (R24h e QFCA), no entanto, auxiliaram na compreensão do perfil/padrão alimentar dos participantes do Grupo Viver Bem a Nova Idade.

O consumo de alimentos do grupo dos pães, cereais e massas, das frutas, leguminosas e leite e derivados foi inferior ao recomendado pelo Ministério da Saúde (BRASIL, 2006; 2014). A quantidade de porções consumidas de pães, massas, cereais e tubérculos está fortemente associada com o valor calórico da dieta, uma vez que esses itens são calóricos e, no Brasil, fazem parte do padrão alimentar da população. Esses resultados contribuem com a justificativa do baixo aporte calórico da dieta dos participantes desta pesquisa. Além disso, é importante mencionar o predomínio do consumo de itens refinados, quando comparado aos integrais, indo na contramão às recomendações do Ministério da Saúde para uma alimentação saudável. As leguminosas e os laticínios são importantes itens alimentares fontes de proteínas e micronutrientes, como ferro e cálcio. A alimentação saudável dos adultos e, em especial, dos idosos, é aquela que respeita os hábitos alimentares e culturais, que são provenientes de sistemas alimentares sustentáveis, que seja diversificada, contando com a presença de todos os grupos alimentares, em quantidade suficiente para proporcionar aporte nutricional em consonância com as recomendações (BRASIL, 2006, 2014).

De acordo com a segunda edição do Guia Alimentar para a População Brasileira, a base da alimentação deve ser composta de alimentos in natura ou minimamente processados, dando preferência aos alimentos de origem vegetal, como frutas e verduras (BRASIL, 2014). Tais alimentos apresentam efeito protetor por conter compostos bioativos com atividade antioxidante, que também atuam na supressão da inflamação e redução do risco de doenças cardiovasculares (BRASIL, 2006; GIANFREDI et al., 2019).

O consumo médio de hortaliças pelos participantes desta pesquisa atendeu às recomendações feitas pelo Ministério da Saúde do Brasil, sendo o consumo superior por adultos maduros, quando comparado com os idosos. O consumo de frutas, no entanto, foi abaixo de três porções/dia, indicando necessidade de estímulo ao consumo desse grupo de alimentos, uma vez que o baixo consumo de frutas também foi associado à presença de sintomas depressivos, por exemplo (MIHRSHANI; DOBSON; MISHRAI, 2015), além da relação direta com a baixa ingestão de compostos bioativos e fibras (BRASIL, 2014; GIANFREDI et al., 2019).

Editora Unijuí - Revista Contexto \& Saúde - ISSN 2176-7114 - v. 21, n. 43, jul./set. 2021 
Tabela 3 - Comparação do consumo em porções dos itens dos grupos alimentares pelos idosos participantes do Grupo Viver Bem a Nova Idade, com as recomendações gerais para idosos do Guia Alimentar para População Brasileira

\begin{tabular}{|c|c|c|c|c|}
\hline \multirow{2}{*}{$\begin{array}{l}\text { Grupo de } \\
\text { Alimentos }\end{array}$} & \multirow{2}{*}{ Itens } & \multicolumn{2}{|c|}{ Consumo (Porções²/dia) } & \multirow{2}{*}{ Ref. ${ }^{\mathrm{a}, \mathrm{t}}$} \\
\hline & & Adultos maduros ${ }^{1}$ & Idosos $^{3}$ & \\
\hline \multirow{5}{*}{$\begin{array}{l}\text { Pães, cereais } \\
\text { e massas }\end{array}$} & $\begin{array}{l}\text { Arroz polido, macarrão conven- } \\
\text { cional e pão francês }\end{array}$ & 2,4 & 3,4 & \multirow{5}{*}{$6^{4}$} \\
\hline & Arroz, macarrão e pão integral & 0,8 & 0,5 & \\
\hline & Biscoito salgado & 0 & 1,0 & \\
\hline & Biscoito recheado & 0 & 0 & \\
\hline & Total & 3,2 & 4,9 & \\
\hline \multirow{3}{*}{ Hortaliças } & Folhosas (verduras) & 2,3 & 2,0 & \multirow{3}{*}{$\geq 3$} \\
\hline & Não folhosas (legumes) & 2,7 & 1,5 & \\
\hline & Total & 5,0 & 3,5 & \\
\hline Frutas & Frutas & 1,5 & 2,3 & $\geq 3$ \\
\hline Leguminosas & $\begin{array}{l}\text { Grãos em geral (feijão, soja e } \\
\text { ervilha) }\end{array}$ & 0,4 & 0,6 & 1 \\
\hline \multirow{4}{*}{$\begin{array}{l}\text { Leites e deri- } \\
\text { vados }\end{array}$} & Leite integral & 0,2 & 0,5 & \multirow{4}{*}{$\geq 3$} \\
\hline & $\begin{array}{l}\text { Leite semidesnatado e desna- } \\
\text { tado }\end{array}$ & 0,4 & 0,3 & \\
\hline & $\begin{array}{l}\text { logurte e queijo minas tipo fres- } \\
\text { cal }\end{array}$ & 1,1 & 0,9 & \\
\hline & Total & 1,7 & 1,7 & \\
\hline \multirow{6}{*}{$\begin{array}{l}\text { Peixes, car- } \\
\text { nes e deriva- } \\
\text { dos }\end{array}$} & Peixe & 0,1 & 0,2 & \multirow{6}{*}{1} \\
\hline & Carne de boi & 0,4 & 0,3 & \\
\hline & Carne de porco & 0,1 & 0,1 & \\
\hline & Carne de frango & 0,5 & 0,3 & \\
\hline & Embutidos & 0,2 & 0,2 & \\
\hline & Total & 1,2 & 1,1 & \\
\hline $\begin{array}{l}\text { Açúcares e } \\
\text { doces }\end{array}$ & Doces & 0,2 & 0,4 & $<1$ \\
\hline $\begin{array}{l}\text { Óleos e gor- } \\
\text { duras }\end{array}$ & Banha e gordura vegetal & 0,3 & 0,2 & $<1$ \\
\hline \multirow{3}{*}{ Bebidas } & Café com açúcar/sem açúcar & 0,7 & 1,0 & \multirow{3}{*}{-} \\
\hline & Chás & 1,2 & 0,6 & \\
\hline & Bebidas alcoólicas & 0,1 & 0,1 & \\
\hline
\end{tabular}

${ }^{1} \mathrm{n}=7$; $^{2}$ Porção é a quantidade de alimento em sua forma usual de consumo expressa em medida caseira, unidade ou forma de consumo, considerando a quantidade média do alimento que deve ser consumida por pessoas sadias para compor uma alimentação saudável (BRASIL, 2006); ${ }^{3} \mathrm{n}=19 ;{ }^{4} \mathrm{O}$ número de porções desse

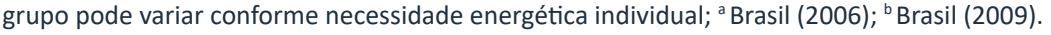

Fonte: Autoria própria. 


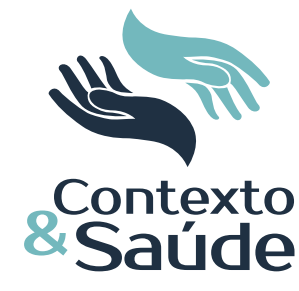

O consumo médio de leites e derivados dos idosos participantes do estudo apresentado na Tabela 3, também foi abaixo do preconizado pelo Ministério da Saúde (BRASIL, 2006, 2009). Indivíduos idosos tendem a reduzir ou parar de consumir leite e seus derivados, posto que esse comportamento pode ser atribuído a diferentes fatores, como a quantidade de gordura nesses alimentos e/ ou ao desenvolvimento de intolerância à lactose. Esse resultado está em acordo com a tendência de se reduzir a aquisição de lacticínios apresentada pela população brasileira. Em 2002, a quantidade per capita anual de laticínios era de 49,9 kg; já em 2018 foi de 32,2 kg (IBGE, 2020). Um estudo de coorte envolvendo 1.215 mulheres suíças após a menopausa, indica que os benefícios do consumo de vegetais para a manutenção da densidade mineral óssea parecem diminuir quando há consumo insuficiente de cálcio e laticínios (LANYAN et al., 2020).

O consumo do grupo dos doces está adequado, considerando que a recomendação de ingestão máxima é de uma porção por dia. Segundo os dados da POF de 2017-2018, o teor médio de açúcar da alimentação do brasileiro corresponde a 9,9\% da energia total disponível (IBGE, 2020), contra um máximo recomendado de $10 \%$ para a população adulta. Assim como o grupo dos doces, o grupo dos óleos e gorduras está dentro do esperado, uma vez que a recomendação para idosos é de, no máximo, uma porção por dia. Além da quantidade total de gorduras da dieta, o perfil de ácidos graxos é extremamente importante para determinar o potencial aterogênico das gorduras (FAO/WHO, 2009). A POF de 2017-2018 indicou contribuição de 10,9\% do teor calórico total para os óleos vegetais e $0,7 \%$ para gordura animal (IBGE, 2020).

\section{Análise do perfil antropométrico}

Segundo estudo realizado com participantes com idade igual ou superior a 60 anos da Pesquisa de Orçamentos Familiares (POF) 2008/2009 (IBGE, 2011), a média do IMC de idosos no Estado de Minas Gerais foi de $25,43 \mathrm{~kg} / \mathrm{m}^{2}$. A média de IMC encontrada nesse estudo foi de $26,8 \pm 6,2 \mathrm{~kg} / \mathrm{m}^{2}$ e $27,6 \pm 7,2 \mathrm{~kg} / \mathrm{m}^{2}$ para adultos maduros e idosos, respectivamente. A avaliação antropométrica demonstrou elevada frequência acima de $40 \%$ dos participantes adultos maduros obesos e idosos com sobrepeso. Além disso, três idosos foram classificados como de baixo peso, sendo indicativo de atenção para desnutrição (Tabela 4).

Mais da metade dos participantes apresentaram risco para doenças cardiovasculares a partir da avaliação da circunferência de cintura. Apesar da elevada frequência de idosos com acúmulo de gordura na região abdominal, a avaliação da circunferência de panturrilha indicou 36,8\% e 5,3\% de idosos com atenção para redução da massa muscular e em risco para sarcopenia, respectivamente (Tabela 4).

Com o envelhecimento observa-se alterações na composição corporal, como o aumento de gordura corporal, especialmente com o acúmulo de gordura na região abdominal e a diminuição da massa magra (PINZÓN-RÍOS, 2019). Essas alterações possibilitam o desenvolvimento de obesidade sarcopênica, condição na qual a redução de massa magra coexiste com excesso de gordura corporal. Todos os idosos que apresentaram circunferência de panturrilha indicativa de atenção para redução de massa muscular ou risco para sarcopenia, no entanto, foram

Editora Unijuí - Revista Contexto \& Saúde - ISSN 2176-7114 - v. 21, n. 43, jul./set. 2021 
classificados como de baixo peso ou eutróficos. Para uma conclusão mais precisa seria necessária a adoção de outros parâmetros antropométricos relacionados ao diagnóstico da sarcopenia.

Tabela 4 - Classificação do estado nutricional, do risco para doenças cardiovasculares e da redução de massa muscular como indicativo de síndrome sarcopênica nos participantes do Grupo Viver Bem a Nova Idade, Alfenas/MG, 2018

\begin{tabular}{lcc}
\hline \multirow{2}{*}{ Parâmetros e classificações } & \multicolumn{2}{c}{ Frequência \% (n) } \\
\hline IMC & Adultos maduros & Idosos \\
$\quad$ Baixo peso & 0 & $15,8(3)$ \\
Peso adequado/eutrofia & $57,1(4)$ & $36,8(7)$ \\
Sobrepeso & 0 & $47,4(9)$ \\
Obesidade & $42,9(3)$ & $\mathrm{NA}$ \\
& & \\
Circunferência de cintura & & $26,3(5)$ \\
Sem risco cardiovascular & $42,9(3)$ & $89,5(17)$ \\
Com risco cardiovascular & $57,1(4)$ & \\
& & \\
Circunferência de panturrilha & & $57,9(11)$ \\
Adequado & NA & $5,3(1)$ \\
Atenção para redução de massa muscular & & \\
Risco para sarcopenia & & \\
\hline
\end{tabular}

Legenda: NA: não se aplica.

Fonte: Autoria própria.

Associado a essa informação, dado que grande parte dos participantes da ação extensionista apresentou sobrepeso (Tabela 4), existe o risco do desenvolvimento de obesidade sarcopênica, condição na qual a redução de massa magra coexiste com excesso de gordura corporal (DENIZ et al., 2020).

A partir da análise da caracterização dos participantes do Projeto de Extensão "Grupo Viver Bem a Nova Idade", foi possível estabelecer alguns temas norteadores das oficinas de educação alimentar e nutricional, visando à promoção da saúde dos participantes: I) promoção de alimentação saudável, seguindo as recomendações da atual edição do Guia Alimentar para a População Brasileira que incentiva o consumo de alimentos in natura e minimamente processados de origem vegetal, a fim de aumentar o aporte de fibra alimentar e a densidade de minerais e vitaminas da dieta, por meio do consumo de frutas, hortaliças, leguminosas e cereais integrais; II) como opção de alimento in natura de origem animal, estimular o consumo de leite, e, esporadicamente, de alguns de seus derivados processados, como queijo branco e coalhada, a fim de se atingir o consumo médio de três porções ao dia e adequar a ingestão de cálcio e vitamina $D$, além de reforçar a importância da exposição ao Sol, preferencialmente entre as 8 e 10 horas da manhã ou após as 16 horas, por período entre 15 a 30 minutos ao dia; III) estimular a redução do consumo de alimentos processados e evitar os ultraprocessados, a fim de adequar a ingestão de ácidos graxos saturados, trans e sódio; IV) conscientizar os participantes sobre a importância de se adotar um estilo de vida ativo, incluindo a busca pela prática monitorada de atividade física para con- 
tribuir com a manutenção da massa magra, e, em especial, evitar dietas restritivas para perda de peso, sem o acompanhamento de um profissional de saúde.

\section{CONCLUSÃO}

Os participantes da ação de EAN em grupo avaliados referiram renda mensal e escolaridade superior à média da população brasileira, elevada frequência de inadequação quanto à distribuição dos macronutrientes, em especial de lipídeos; elevada frequência de ingestão aumentada de ácidos graxos saturados, trans e de sódio; elevado número de participantes consumindo fibras alimentares e cálcio abaixo do preconizado; e inadequação para a ingestão de vitamina D. Houve predomínio de sobrepeso, risco cardiovascular e frequência acima de $35 \%$ de situação de atenção para a redução e massa muscular. O desenvolvimento desta pesquisa possibilitou compreender a situação dos participantes da pesquisa a partir de sua visão multidimensional, o que contribuiu com a organização e seleção de temas para as futuras ações de educação alimentar e nutricional.

\section{REFERÊNCIAS}

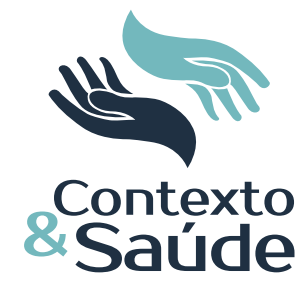

AHA. American Heart Association. Dietary fats and cardiovascular disease: a presidential advisory from the American Heart Association. AHA Journal, v. 136, p. e1-e23, 2017. DOI: 10.1161/CIR.0000000000000510

BARROS, M. B. A. et al. Social inequalities in health behaviors among Brazilian adults: National Health Survey, 2013. Int J Equity Health, v. 15, n. 1, p. 148, 2016.

BEDESCHI, L. B. et al. Grupo operativo: estratégia de aprendizagem na educação nutricional em diabetes. Repenf-Rev. Par. Enferm., v. 1, n. 1, p. 47-58, 2018.

BRASIL. Ministério da Saúde (MS). Guia alimentar para a população brasileira - promovendo a alimentação saudável. 1. ed. Brasília: Ministério da Saúde, 2006. 210 p.

BRASIL. Ministério da Saúde. Secretaria de Atenção à Saúde. Departamento de Atenção Básica. Alimentação saudável para a pessoa idosa: um manual para profissionais de saúde. Brasília: Ministério da Saúde, 2009. 36 p.

BRASIL. Ministério da Saúde. Coordenação Geral de Alimentação e Nutrição. Departamento de Atenção Básica. Orientações para a coleta e análise de dados antropométricos em serviços de saúde: norma técnica do sistema de vigilância alimentar e nutricional Sisvan. Brasília: Ministério da Saúde, 2011.

BRASIL. Ministério da Saúde. Secretaria de Atenção à Saúde. Departamento de Atenção Básica. Guia alimentar para a população brasileira. 2. ed. Brasília: Ministério da Saúde, 2014. 156 p.

BRASIL. Ministério da Saúde. Departamento de Atenção Básica. Caderneta de Saúde da Pessoa Idosa. Brasília: Ministério da Saúde, 2017.

BRASIL. Ministério da Saúde. Vigitel Brasil 2018: vigilância de fatores de risco e proteção para doenças crônicas por inquérito telefônico: estimativas sobre frequência e distribuição sociodemográfica de fatores de risco e proteção para doenças nas capitais dos 26 Estados brasileiros e no Distrito Federal em 2018. Brasília: Ministério da Saúde, 2019. $132 \mathrm{p}$.

CANO-IBÁÑEZ, N. C. et al. Diet quality and nutrient density in subjects with metabolic syndrome: Influence of socioeconomic status and lifestyle factors. A cross-sectional assessment in the Predimed-Plus study. Clinical Nutrition, v. 39, p. 1.161-1.173, 2020.

DARMON, N.; DREWNOWSKI, A. Contribution of food prices and diet cost to socioeconomic disparities in diet quality and health: a systematic review and analysis. Nutr Rev., v. 73, n. 10, p. 643-60, 2015.

Editora Unijuí - Revista Contexto \& Saúde - ISSN 2176-7114 - v. 21, n. 43, jul./set. 2021 
DENIZ, O. et al. Role of Ultrasonography in Estimating Muscle Mass in Sarcopenic Obesity. JPEN J Parenter Enteral Nutr. [Epub ahead of print], 2020.

ESPEN. European Society for Clinical Nutrition and Metabolism. In: VOLTERT, D. et al. ESPEN guideline on clinical nutrition and hydration in geriatrics. Clinical Nutrition, v. 38, p. 10-47, 2019.

FAO/WHO. Joint FAO/WHO Expert Consultation. In: ELMADFA, I.; KORNSTEINER, M. Fats and fatty acid requirements for adults. FATS AND FATTY ACIDS IN HUMAN NUTRITION. Annals of Nutrition and Metabolism, Geneva, 2009. p. 56-75. Disponível em: https:// www.karger.com/Journal/Issue/250361. Acesso em: 10 maio 2020.

GABE, K. T.; JAIME, P. C. Dietary practices in relation to the Dietary guidelines for the Brazilian population: associated factors among Brazilian adults, 2018. Epidemiol. Serv. Saude, Brasília, v. 29, n. 1, 2020.

GIANFREDI, V. et al. Rectal Cancer: 20\% Risk Reduction Thanks to Dietary Fibre Intake. Systematic Review and Meta-Analysis. Nutrients, v. 11, n. 7, 2019.

IOM. Institute of Medicine. Dietary reference intakes for energy, carbohydrate, fiber, fat, fatty acids, cholesterol, protein, and amino acids. Washington, DC: The National Academies Press, 2005. 1.358 p. Disponível em: https://www.nap.edu/catalog/10490/ dietary-reference-intakes-for-energy-carbohydrate-fiber-fat-fatty-acids-cholesterol-protein-and-amino-acids. Acesso em: 10 maio 2020.

IOM. Institute of Medicine. Dietary reference intakes for calcium and vitamin D. Washington, DC: The National Academies Press, 2011. 1.132 p. Disponível em: https://www.nap. edu/catalog/13050/dietary-reference-intakes-for-calcium-and-vitamin-d. Acesso em: 10 maio 2020.

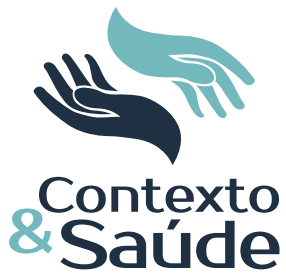

OM. Institute of Medicine. Dietary reference intakes for sodium and potassium. Washington, DC: The National Academies Press, 2019. 594 p. Disponível em: https://www.nap. edu/catalog/25353/dietary-reference-intakes-for-sodium-and-potassium. Acesso em: 10 maio 2020.

IBGE. Instituto Brasileiro de Geografia e Estatística. Coordenação de Trabalho e Rendimento. Pesquisa de orçamentos familiares 2008-2009: análise do consumo alimentar pessoal no Brasil. Rio de Janeiro, 2011. 150 p.

IBGE. Instituto Brasileiro de Geografia e Estatística. Pesquisa de orçamentos familiares: 2017, 2018: avaliação nutricional da disponibilidade domiciliar de alimentos no Brasil. Rio de Janeiro: IBGE, 2020. 56 p. Disponível em: https://biblioteca.ibge.gov.br/index.php/ biblioteca-catalogo?view=detalhes\&id=2101704. Acesso em: 11 maio 2020.

LANYAN, A. et al. Postmenopausal women with osteoporosis consume high amounts of vegetables but insufficient dairy products and calcium to benefit from their virtues: the CoLaus/OsteoLaus cohort. Osteoporosis International, v. 31, p. 875-886, 2020.

MIHRSHANI, S.; DOBSON, A. J.; MISHRAI, G. D. Fruit and vegetable consumption and prevalence and incidence of depressive symptoms in mid-age women: results from the Australian longitudinal study on women's health. European Journal of Clinical Nutrition, v. 69, n. 1, p. 585-91, 2015.

OMS. Organização Mundial de Saúde. Obesity: preventing and managing the global epidemic. Geneva: World Health Organization, 1998.

OMS. Organização Mundial da Saúde. Relatório Mundial de Envelhecimento e Saúde. 2015. Disponível em: https://sbgg.org.br/wp-content/uploads/2015/10/OMS-ENVELHECIMENTO-2015-port.pdf. Acesso em: 12 fev. 2020.

PACHECO, M. Tabela de equivalentes, medidas caseiras e composição química dos alimentos. Rio de Janeiro: Editora Rubio, 2011. 688 p.

PINZÓN-RÍOS, I. D. Loss of Muscle Mass Induced by Aging. Rev. Cienc. Salud, Bogotá, v. 17, n. 2, p. 223-244, ago. 2019.

QUEIROZ, F. L. N. et al. Eating competence associated with food consumption and health outcomes among Brazilian adult population. Nutrients, v. 12, n. 3.218, 2020.

SOEIRO, R. L. et al. Educação em saúde em grupo no tratamento de obesos grau III: um desafio para os profissionais de saúde. Rev. Bras. Educ. Med., Brasília, v. 43, n. 1, supl. 1, p. 681-691, 2019. 
SCHALAFF, R. A. et al. Effects of a group-based behavioral intervention on dietary behaviors in older adults. Journal of Aging and Health, v. 30, n. 1, p. 105-17, 2018.

VINCHA, K. R. R.; BOGUS, C. M.; CERVATO-MANCUSO, A. M. Possibilidades de atuação profissional em grupos educativos de alimentação e nutrição. Interface, Botucatu, v. 24, e190028, 2020.

YANG, A. et al. The effect of vitamin D on sarcopenia depends on the level of physical activity in older adults. Journal of Cachexia, Sarcopenia and Muscle, p. 1-12, 2020. DOI: 10.1002/jcsm.1245 